\title{
Metabolic Regulation of Pluripotent Stem Cell Expansion, Differentiation and Reprogramming
}

Yan Li ${ }^{1}$ and Shang-Tian Yang ${ }^{2 *}$

${ }^{1}$ Department of Chemical and Biomedical Engineering, Florida State University, USA

${ }^{2}$ William G Lowrie Department of Chemical and Biomolecular Engineering, The Ohio State University, USA

In recent years, metabolic plasticity of human Pluripotent Stem Cells (hPSCs), including human Embryonic Stem Cells (hESCs) and induced Pluripotent Stem Cells (iPSCs), emerges as an important area to regulate hPSC expansion, differentiation, and the iPSC reprogramming $[1,2]$. PSCs have unique energy and biosynthetic requirements and utilize predominantly glucose through glycolysis rather than Oxidative Phosphorylation (OXPHOS) at undifferentiated state [3]. Upon differentiation, the metabolic pathway is observed to shift from glycolysis to OXPHOS [2]. The reverse process is also observed during the reprogramming of somatic cells to iPSCs, where the metabolic pathway shifts from OXPHOS to glycolysis when the cells gain pluripotency [4]. Metabolic pathways of PSCs (e.g. glucose metabolism, mitochondrial function, redox status, etc.) can be regulated by environmental conditions such as oxygen and the biomolecules that act in the intermediate steps of glycolysis and/or OXPHOS [5]. Understanding the metabolic status during different stages of PSC development can benefit various aspects of PSC engineering in expansion, differentiation, and reprogramming.

\section{Expansion and Maintenance of hPSC in Undifferentiated State}

Metabolic modulation has been used to develop new culture systems for undifferentiated hPSCs. For example, modulation of mitochondria function using antimycin A, which decreases ATP production from OXPHOS and Reactive Oxygen Species (ROS) formation, can support undifferentiated hPSC propagation by stimulating the metabolic shift from OXPHOS to glycolysis [6]. The pluripotent marker OCT4 affects several target genes (e.g. ATP5D) associated with energy metabolism which are in favor of glycolysis [1]. Various new hPSC culture systems have been developed in recent years, including defined serum-free media (e.g., mTESR, StemPro, and E8) and synthetic substrates (e.g., synthemax) $[7,8]$. How the subtle differences in these culture conditions affect energy balance and the differentiation efficiency of hPSCs needs to be explored.

\section{IPSC Reprogramming}

Metabolic plasticity of PSCs has been recently demonstrated in the method development of iPSC reprogramming [4]. The somatic cells switch the metabolism from OXPHOS to the enhanced glycolysis and become pluripotent when reprogramed with pluripotent genes and/or small molecules $[9,10]$. Compared to early passages of iPSCs, the levels of metabolites from late passages of iPSCs were found more close to those of ESCs. The slow transition in the metabolic profile changes also suggested the effect of "metabolic memory" during reprogramming [9]. Consequently, the somatic cells with a higher glycolytic and lower OXPHOS capacity showed higher reprogramming efficiency $[4,9]$. Therefore, promotion of glycolic metabolism by drugs, hypoxia, or supplementation of glycolic intermediates (e.g. PS48, a small molecule activator of 3'-phosphoinositide-dependent kinase-1 (PDK1)) has been found to augment the reprogramming efficiency, while inhibition of glycolysis and/or stimulation of OXPHOS impaired reprogramming [9-11].

\section{Specific Lineage Differentiation: Monolayer or Embryoid Body (EB)?}

Most current hPSC differentiation protocols either use the formation of $\mathrm{EBs}$ or through monolayer induction by growth factors. Higher differentiation efficiency has been observed for some monolayer protocols for several lineages (cardiomyocytes, hematopoietic progenitors, etc) compared to EB protocols $[12,13]$. Due to the differences in oxygen and nutrient transport in EBs compared to monolayer cultures, distinct different metabolic fluxes were observed during spontaneous differentiation with EBs displaying a stronger lactogenic pattern [14]. However, the energy needs for lineage-specific differentiation may be different from spontaneous differentiation. Studying the effect of 3-D organization on metabolic fluxes during lineage-specific differentiation would be required.

\section{The Case of Neural Differentiation}

Although neural stem/progenitor cells can be derived from hPSCs, little is known about the bioenergetic requirements during this cellular process. Contrary to the increased utilization of OXPHOS during hPSC differentiation, results also have been reported for the shift from OXPHOS to glycolysis during neural differentiation of hPSCs $[15,16]$. The conflicting results may be due to the variations in neural differentiation protocols, the culture conditions, and the stage of differentiation. For example, proliferating neural progenitor cells showed higher levels of glycolysis compared to neural stem cells [2]. Evaluation of metabolic shift for differentiation into different subtypes of neural cells has direct impact in PSC engineering. Most neural differentiation protocols from hPSCs are lengthy. For example, the derivation of Oligodendrocyte Progenitor Cells (OPCs) from hESCs needs 41 days in the production process involving both suspension and adherent cultures [17]. The failure of the production process will cause huge economical loss due to the scale of production. The ability to predict the differentiation outcome by monitoring the metabolic activity will greatly benefit the production of hPSC-derived OPCs.

*Corresponding author: Shang-Tian Yang, William G. Lowrie Department of Chemical and Biomolecular Engineering, The Ohio State University, USA, Tel: 614292-6611; Fax: 614-292-3769; E-mail: yang.15@osu.edu

Received November 20, 2013; Accepted November 22, 2013; Published November 25, 2013

Citation: Yan Li, Yang ST (2013) Metabolic Regulation of Pluripotent Stem Cell Expansion, Differentiation and Reprogramming. J Tissue Sci Eng 4: e125. doi:10.4172/2157-7552.1000e125

Copyright: (c) 2013 Yan Li, et al. This is an open-access article distributed under the terms of the Creative Commons Attribution License, which permits unrestricted use, distribution, and reproduction in any medium, provided the original author and source are credited. 
Citation: Yan Li, Yang ST (2013) Metabolic Regulation of Pluripotent Stem Cell Expansion, Differentiation and Reprogramming. J Tissue Sci Eng 4: e125. doi:10.4172/2157-7552.1000e125

\section{The Case of Cardiomyocyte Differentiation}

Cardiomyocyte differentiation has been significantly improved in recent years due to stage-wise growth factor induction or modulation of Wnt signaling $[12,18,19]$. However, the robustness of the process relies on the status of the cell population before the growth factor induction, even though the cells do express high levels of pluripotent markers. The ability to detect the subtle differences in hPSCs contributing to differentiation variations is needed for robust production of hPSCderived cardiomyocytes. The endogenous ROS level, which is promoted in high glucose medium, was found to enhance cardiomyocyte differentiation from PSCs [20]. In addition, downstream purification is also critical to the isolation of cardiomyocytes because the differentiation efficiency is low (20-50\%) in most of the protocols. Based on distinct metabolic flow, it has been demonstrated that hPSCderived cardiomyocytes can be enriched under glucose-depleted and lactate abundant conditions [21]. Providing the medium that can induce adult-like energy metabolism was also found to accelerate the maturation of human iPSC-derived cardiomyocytes, which usually display embryonic heart cell function [22].

\section{Current Challenges and Perspectives}

Although the metabolic shift correlates with the status during PSC expansion, differentiation, and iPSC reprogramming, applications of metabolic regulation in hPSC-derived cell production are still illusive and needs further studies and better understanding of the effects of various metabolites and substrates. Glucose and oxygen are the two most important substrates in cell metabolism, and their concentrations in the culture media can be used to modulate metabolic status of cells. Design the feeding regime and the exposure to hypoxia condition can help to regulate the cellular composition. In addition, small molecules affecting the mitochondrial function and glycolysis/OXPHOS pathways can also be used to modulate PSC fate decisions. Understanding the metabolic plasticity can help design the process in PSC engineering in the following aspects: 1) developing well-defined culture conditions for hPSCs; 2) process monitoring based on changes in the metabolic status of the cells; 3 ) developing novel downstream separation process for purifying the cells of specific lineages differentiated from hPSCs. These developments depend on if the following questions can be answered: 1) Can metabolic status be used to predict the specific lineage differentiation efficiency? 2) Can metabolic status be used to modulate the composition of cell population? 3) Can metabolic regulation be used for process integration of iPSC reprogramming, expansion, and differentiation? Taken together, translation the findings in metabolic plasticity of hPSCs to the manufacturing process should accelerate the advancements in hPSC-derived cell production for drug screening, disease modeling, and tissue engineering.

\section{References}

1. Folmes CD, Dzeja PP, Nelson TJ, Terzic A (2012) Metabolic plasticity in stem cell homeostasis and differentiation. Cell Stem Cell 11: 596-606.

2. Shyh-Chang N, Daley GQ, Cantley LC (2013) Stem cell metabolism in tissue development and aging. Development 140: 2535-2547.

3. Varum S, Rodrigues AS, Moura MB, Momcilovic O, Easley CA 4th, et al. (2011) Energy metabolism in human pluripotent stem cells and their differentiated counterparts. PLoS One 6: e20914.

4. Zhang J, Nuebel E, Daley GQ, Koehler CM, Teitell MA (2012) Metabolic regulation in pluripotent stem cells during reprogramming and self-renewal. Cell Stem Cell 11: 589-595.

5. Vacanti NM, Metallo CM (2013) Exploring metabolic pathways that contribute to the stem cell phenotype. Biochim Biophys Acta 1830: 2361-2369.
6. Varum S, MomciloviÄ $\mathrm{O}$, Castro C, Ben-Yehudah A, Ramalho-Santos J, et al. (2009) Enhancement of human embryonic stem cell pluripotency through inhibition of the mitochondrial respiratory chain. Stem Cell Res 3: 142-156.

7. Melkoumian Z, Weber JL, Weber DM, Fadeev AG, Zhou Y, et al. (2010) Synthetic peptide-acrylate surfaces for long-term self-renewal and cardiomyocyte differentiation of human embryonic stem cells. Nat Biotechnol 28: 606-610.

8. Chen G, Gulbranson DR, Hou Z, Bolin JM, Ruotti V, et al. (2011) Chemically defined conditions for human iPSC derivation and culture. Nat Methods 8: 424 429 .

9. Panopoulos AD, Yanes O, Ruiz S, Kida YS, Diep D, et al. (2012) The metabolome of induced pluripotent stem cells reveals metabolic changes occurring in somatic cell reprogramming. Cell Res 22: 168-177.

10. Zhu S, Li W, Zhou H, Wei W, Ambasudhan R, et al. (2010) Reprogramming of human primary somatic cells by OCT4 and chemical compounds. Cell Stem Cell 7: 651-655.

11. Yoshida Y, Takahashi K, Okita K, Ichisaka T, Yamanaka S (2009) Hypoxia enhances the generation of induced pluripotent stem cells. Cell Stem Cell 5: 237-241.

12. Laflamme MA, Chen KY, Naumova AV, Muskheli V, Fugate JA, et al. (2007) Cardiomyocytes derived from human embryonic stem cells in pro-survival factors enhance function of infarcted rat hearts. Nat Biotechnol 25: 1015-1024.

13. Niwa A, Heike T, Umeda K, Oshima K, Kato I, et al. (2011) A novel serum-free monolayer culture for orderly hematopoietic differentiation of human pluripotent cells via mesodermal progenitors. PLoS One 6: e22261.

14. Sepúlveda DE, Andrews BA, Papoutsakis ET, Asenjo JA (2010) Metabolic flux analysis of embryonic stem cells using three distinct differentiation protocols and comparison to gene expression patterns. Biotechnol Prog 26: 1222-1229.

15. Birket MJ, Orr AL, Gerencser AA, Madden DT, Vitelli C, et al. (2011) A reduction in ATP demand and mitochondrial activity with neural differentiation of human embryonic stem cells. J Cell Sci 124: 348-358.

16. Zhang J, Khvorostov I, Hong JS, Oktay Y, Vergnes L, et al. (2011) UCP2 regulates energy metabolism and differentiation potential of human pluripotent stem cells. EMBO J 30: 4860-4873.

17. Li Y, Gautam A, Yang J, Qiu L, Melkoumian Z, et al. (2013) Differentiation of oligodendrocyte progenitor cells from human embryonic stem cells on vitronectin-derived synthetic Peptide acrylate surface. Stem Cells Dev 22: 1497-1505.

18. Xu C, Police S, Hassanipour M, Li Y, Chen Y, et al. (2011) Efficient generation and cryopreservation of cardiomyocytes derived from human embryonic stem cells. Regen Med 6: 53-66.

19. Lian X, Hsiao C, Wilson G, Zhu K, Hazeltine LB, et al. (2012) Robust cardiomyocyte differentiation from human pluripotent stem cells via temporal modulation of canonical Wnt signaling. Proc Natl Acad Sci U S A 109: E18481857.

20. Crespo FL, Sobrado VR, Gomez L, Cervera AM, McCreath KJ (2010) Mitochondrial reactive oxygen species mediate cardiomyocyte formation from embryonic stem cells in high glucose. Stem Cells 28: 1132-1142.

21. Tohyama S, Hattori F, Sano M, Hishiki T, Nagahata Y, et al. (2013) Distinct metabolic flow enables large-scale purification of mouse and human pluripotent stem cell-derived cardiomyocytes. Cell Stem Cell 12: 127-137.

22. Kim C, Wong J, Wen J, Wang S, Wang C, et al. (2013) Studying arrhythmogenic right ventricular dysplasia with patient-specific iPSCs. Nature 494: 105-110. 